\title{
VALIDACIÓN DE UN MÉTODO VOLTAMÉTRICO PARA LA CUANTIFICACIÓN DE MANCOZEB EN CEBOLLA CABEZONA (Allium cepa $L$.)
}

\section{VALIDATION OF A VOLTAMMETRIC METHOD FOR THE ESTIMATION OF MANCOZEB IN BULB ONION (Allium cepa L.)}

\author{
Edwin R. Martínez ${ }^{1}$, Mónica Y. Dotor ${ }^{2}$, Sandra P. Chaparro ${ }^{3 *}$
}

\begin{abstract}
${ }^{1}$ Químico de Alimentos, Universidad Pedagógica y Tecnológica de Colombia -UPTC-, Tunja, e-mail: edwinricardo.martinez@ uptc.edu.co; ${ }^{2}$ Ingeniera Agrónoma UPTC. M.Sc. Fisiología Vegetal UPTC- Tunja. Estudiante de Doctorado en Ciencias Agrarias Universidad Nacional de Colombia (UNAL), docente Facultad de Ciencias Agropecuarias UPTC-Tunja, integrante Grupo Manejo Biológico de Cultivos UPTC, e-mail: monica.dotor@uptc.edu.co; ${ }^{3}$ Química de Alimentos, Especialista y Magister en Ciencia y Tecnología de Alimentos UNAL. Docente Facultad de Ciencias, integrante Grupo de Investigación en Química Ambiental UPTC, e-mail: patricia.chaparro@uptc.edu.co*dirección para correspondencia
\end{abstract}

Rev. U.D.C.A Act. \& DIv. Cient. 17/2): 607-611, Julio-Diciembre, 2014

\section{RESUMEN}

Se validó un método sensible para la cuantificación de mancozeb en cebolla cabezona. El estudio fue realizado utilizando como electrolito de soporte solución buffer BrittonRobinson. Un electrodo de $\mathrm{Ag} / \mathrm{AgCl}$ fue empleado como referencia y, como electrodos de trabajo, carbón vítreo y platino. El mancozeb presentó dos picos de reducción en $-860 \mathrm{mV}$ y $-110 \mathrm{mV}$, donde el perfil de proceso de reducción es totalmente reversible y el uso de los pulsos permitió la detección de niveles de partes por billón (ppb), después de la optimización de las condiciones voltamétricas. La preparación de la muestra incluyó la extracción sólido líquido con diclorometano. El límite de detección fue de 15,1ppb y el límite de cuantificación fue de 76,5ppb. Los resultados obtenidos fueron contrastados por espectroscopia UV-Vis, permitiendo deducir que el método voltamétrico genera resultados precisos y confiables para la cuantificación de mancozeb en cebolla cabezona.

Palabras clave: Incertidumbre, límite de detección, productos agrícolas, fungicida.

\section{SUMMARY}

A sensitive method for quantification of mancozeb in bulb onion was validated. The study was conducted using as supporting electrolyte Britton-Robinson buffer solution. $\mathrm{Ag} / \mathrm{AgCl}$ electrode was used as reference electrode while the working electrodes were glassy carbon and platinum electrodes. Mancozeb fungicide presented two reduction peaks, in $-860 \mathrm{mV}$ and $-1100 \mathrm{mV}$, with profile of the redox process totally reversible; the use of pulses allowed a detection of ppb levels after the optimization of experimental and voltammetric conditions. Sample preparation included solid liquid extraction with dichloromethane. The detection limit was 15.1ppb and the limit of quantification was 76.5ppb. The results obtained were compared by UV-Vis spectroscopy allows to deduce that the voltammetric method generates accurate and reliable quantification of mancozeb in bulb onion.

Key words: Uncertainty, limit of detection, agricultural products, fungicide.

\section{INTRODUCCIÓN}

El mancozeb es un fungicida de contacto de amplio espectro, que se utiliza como tratamiento preventivo de enfermedades fúngicas, que atacan diversos cultivos (López et al. 2013). Este compuesto tiene una solubilidad limitada en agua, entra en superficiales del suelo, con una vida media de uno a siete días, dependiendo de las condiciones ambientales (Harrison et al. 2013); su principal metabolito producto de la degradación por hidrólisis y por fotólisis es la etilentiourea (ETU) (Garcinuño et al. 2004), analito relacionado con degeneración neural (Harrison et al. 2013), efectos genotóxicos (Dearfield, 1994), carcinogénicos (Valentich et al. 
2006), mutagénicos y teratogénicos (Vettorazzi et al. 1995). Los métodos de cuantificación de este fungicida incluyen cromatografía (Mujawar et al. 2014; Blasco et al. 2004) y espectrofotometría UV-Vis (Paramasivam \& Chandrasekaran, 2013).

La cebolla cabezona (Allium cepa L.) es la hortaliza más consumida en el mundo después del tomate, siendo los mayores productores China, India y Estados Unidos. En Colombia, este alimento es importante por ser un generador de ingresos para los pequeños cultivadores, principalmente, en los departamentos de Boyacá, con un 52\% de la producción del país, seguido de Nariño y Cundinamarca (Ministerio de Agricultura, 2002). La baja sostenibilidad y competitividad relacionadas con la problemática sanitaria y el deterioro del medio ambiente proporciona bajos rendimientos y obliga al uso extensivo de agroquímicos (Sena, 2006). El objetivo de este trabajo fue validar el método electroquímico para la cuantificación de mancozeb en cebolla cabezona blanca y aplicarlo, para analizar residuos en este alimento en el periodo de carencia en producto procedente del Sector de Santa Teresa, Municipio de Tibasosa, departamento de Boyacá.

\section{MATERIALES Y MÉTODOS}

Reactivos: Hidróxido de sodio al 99\%, ácido acético glacial al 99,8\%, ácido bórico al 99\%, ácido fosfórico al 85\%, ácido nítrico al $65 \%$ y cloruro de amonio al $99 \%$, empleados en el estudio, fueron grado analítico, obtenidos de Merck y, el mancozeb al 98\%, de la casa comercial Fluka. El agua utilizada para preparación de reactivos y purgas de materiales fue desionizada con una conductividad menor de 0,05 $\mu \mathrm{S}$ (Sistema Milli-Di ${ }^{\mathrm{TM}}$ y Simplicity).

Equipos: Polarógrafo BAS CV 50W con programa BAS CV 50 w versión 2.0 , que cuenta con un analizador voltamperométrico y un electrodo de carbono vítreo, como electrodo de trabajo; electrodo de referencia $\mathrm{Ag} / \mathrm{AgCl}$ y un electrodo auxiliar de platino, balanza analítica Aventure Ohaus, micropipeta Boeco de 10-100uL, pHmetro Sartorius Scohott CG 842 y espectrofotómetro Genesys 10 .

Soluciones estándar y de trabajo: Se preparó una solución stock de 50ppm de mancozeb y, a partir de ésta, una de $10 \mathrm{ppm}$. Se elaboraron soluciones estándar a diferentes concentraciones, de 50, 100, 150, 200, 250, 300, 350 y 450ppb, para la curva de calibración. El electrolito de soporte usado fue buffer Britton Robinson, el cual, se preparó adicionando, a un balón de $100 \mathrm{~mL}, 0,618 \mathrm{~g}$ de ácido bórico y $0,56 \mathrm{~mL}$ de ácido acético y $0,48 \mathrm{~mL}$ de ácido orto fosfórico, se aforó con agua desionizada y se ajustó a pH 5, con $\mathrm{NaOH} 0,1 \mathrm{~N}$.

Preparación de la muestra: Las muestras de cebolla cabezona para el estudio fueron recolectadas de forma aleatoria, en las que se cubría la totalidad de cultivos del Sector Santa Teresa, Municipio de Tibasosa, en el periodo de carencia, que es el tiempo mínimo (25-30 días), que debe transcurrir entre la última aplicación de un plaguicida y la cosecha del producto, para garantizar que el compuesto aplicado se haya degradado y sus residuos, en el producto cosechado, no superen los límites máximos permisibles del Codex Alimentarius; en el caso de la cebolla, se tomó muestras a los 5, 15 y 25 días, de la última adición del producto. Las muestras se lavaron, se cortaron en finas capas y se secaron a $55^{\circ} \mathrm{C}$; se molió el material seco y se realizó extracción del fungicida con diclorometano, según la metodología de extracción sólido - líquido, propuesta por Qiu \& Nian (2008). Se adicionaron $4 \mathrm{~mL}$ de electrolito de soporte (Britton Robinson $\mathrm{pH}$ 5) y $3 \mathrm{~mL}$ de muestra extraída y se ajustó a pH 4,8. La mezcla obtenida, se transfirió a la celda electroquímica, se programó el equipo, según la optimización previa de los parámetros y se procedió a la medición.

Validación del método: La determinación de mancozeb en cebolla cabezona, se realizó de acuerdo al método de Qiu \& Nian (2008). Se contemplaron las siguientes etapas principales: limpieza del sistema de electrodos, selección de condiciones instrumentales: potencial inicial, potencial final, desoxigenación de la muestra, tiempo de quietud, sensibilidad, amplitud, frecuencia de onda y rango de potencial. Se determinaron los principales atributos del método: límite de detección (DL), límite de cuantificación (QL), precisión, exactitud, sensibilidad e incertidumbre. Para evaluar la reproducibilidad del método, se evalúo con el método colorimétrico, referenciado por Kaur et al. (2011).

Diseño experimental: Para optimizar las condiciones del análisis voltamétrico, se empleó un diseño experimental de cuadrado latino al azar $3 * 3$, con tres repeticiones independientes para cada parámetro evaluado (tiempo de desoxigenación, tiempo de quietud, rango de trabajo, sensibilidad, amplitud de onda y frecuencia de onda). La validación, se evaluó a través de un diseño factorial $2^{k}$; las muestras, se analizaron al azar por triplicado y, en total, se realizaron 64 ensayos, por dos analistas. Las diferencias estadísticas, se determinaron mediante un Análisis de Varianza, utilizando el software estadístico SPSS 18 para Windows.

\section{RESULTADOS Y DISCUSIÓN}

Validación del método voltamétrico: Los parámetros finales establecidos para el método fueron: desoxigenación de la muestra: $5 \mathrm{~min}$, Rango de potencial de trabajo: -600 A $-1300 \mathrm{mV}$, Potencial inicial: $-600 \mathrm{mV}$, Potencial final: $1300 \mathrm{mV}$, Sensibilidad: $100 \mathrm{mS}$, Tiempo de quietud: $5 \mathrm{seg}$, Velocidad de barrido e intervalo de muestreo: $20 \mathrm{mV} / \mathrm{s}$. El compuesto de mancozeb presenta dos señales voltamétricas, las cuales, indican que el proceso de reducción es llevado a cabo en dos 
pasos. La primera señal, se presenta hacia Zn: $-1100 m V$ y, la segunda, hacia $\mathrm{Mn}$ : $-860 \mathrm{mV}$, que son potenciales únicos para estos dos elementos (Figura 1). El pH óptimo de trabajo fue de 5, tanto para el zinc como para el manganeso. $\mathrm{Al}$ analizar los puntos obtenidos en la parte experimental, se pudo constatar que los valores de coeficientes de correlación $r$ para el método evaluado fueron: $M n: y=2 E^{-11} x-9 E^{-09}$, con un coeficiente de correlación de $R^{2}=0,9965$ y Zn:y=2E-12x$3 \mathrm{E}-09$, con un coeficiente de correlación de $\mathrm{R}^{2}=0,9878$, lo que permite garantizar que existe proporcionalidad de la intensidad con respecto a la concentración y asegura que los resultados se ajusten a una ecuación de tipo lineal. La sensibilidad del método, se calculó con la pendiente de la curva de trabajo y quedó establecida para señal de $M n: 2 E^{-11} \mathrm{~A} / \mathrm{ppb}$ y $\mathrm{Zn}: 2 \mathrm{E}^{-12} \mathrm{~A} / \mathrm{ppb}$. La precisión evaluada, como repetibilidad y reproducibilidad, mostraron resultados confiables, pues los coeficientes de variación determinados, en ambos métodos, cumplieron con el criterio de aceptación ( $\geq 5 \%$ ).

Todo el estudio desarrollado permite afirmar que los resultados obtenidos por el método voltamétrico, propuesto para la cuantificación de mancozeb en cebolla cabezona, son confiables, pues demuestran mantener los criterios fundamentales de linealidad, de precisión, de exactitud y de especificidad (Tabla 1). Cabe resaltar, que los límites de detección y de cuantificación son más bajos que los de otros métodos usados, que están expresados en ppm.

Una vez validado el método voltamétrico para la cuantificación de mancozeb, se aplicó a estándares y se verificó la confiabilidad de las mediciones voltamétricas; estos resultados fueron comparados con los obtenidos en el método de espectrofotometría UV-Vis. Las concentraciones determinadas de mancozeb en las muestras y estándares por voltametría fueron superiores a las del método de referencia, es decir,

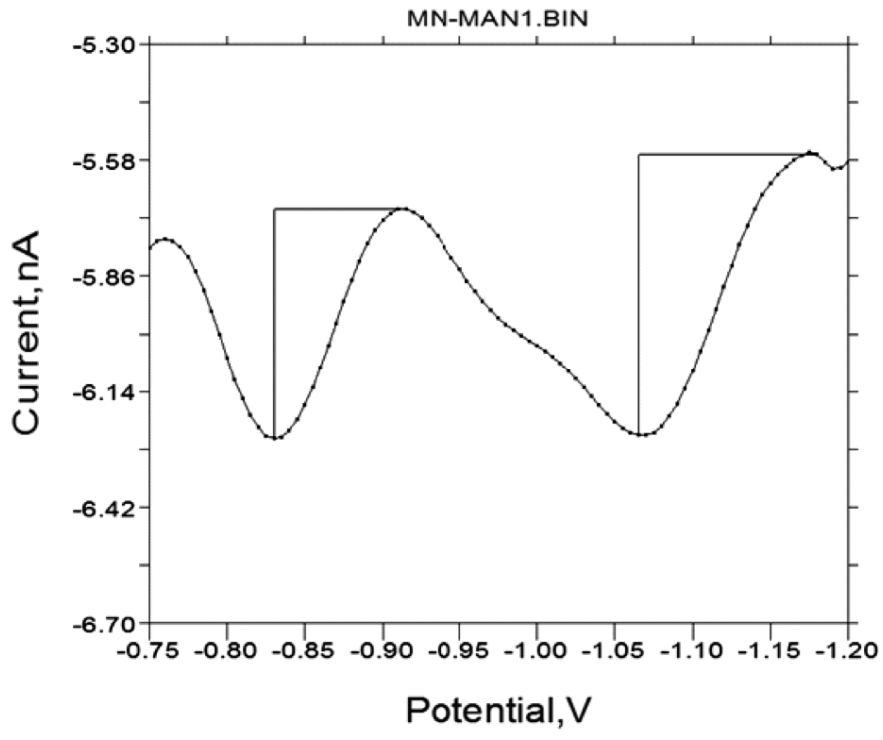

Figura 1. Voltametría lineal para reducción de Zinc $(-860$ mV) y Manganeso (-1100 mV).

Tabla 1. Parámetros validación de mancozeb.

\begin{tabular}{|l|c|}
\hline \multicolumn{1}{|c|}{ ATRIBUTO } & VALOR \\
\hline Nivel de confianza & $95,00 \%$ \\
\hline Límite de detección & $15,087 \mathrm{ppb}$ \\
\hline Límite de cuantificación & $76,536 \mathrm{ppb}$ \\
\hline Sensibilidad & $2 \mathrm{E}-11 \mathrm{l} / \mathrm{ppp}$ \\
\hline Recuperación adicionado alto & $96,126 \%$ \\
\hline Recuperación adicionado bajo & $91,136 \%$ \\
\hline Precisión (reproducibilidad) & $87,65 \%$ \\
\hline Exactitud & $88,39 \%$ \\
\hline Incertidumbre & $\pm 0,2033 \mathrm{ppb}$ \\
\hline
\end{tabular}


el método es más sensible y registra concentraciones más bajas del analito en muestras de cebolla. Esta diferencia, se evaluó por medio de un análisis de varianza de un factor donde se tuvo, como referencia, la concentración de los estándares. No se presentó diferencia en la concentración de los estándares del fungicida, por las dos técnicas de análisis.

Evaluación del periodo de carencia: Durante el periodo de carencia, se observó una disminución del fungicida a través del tiempo, lo que indica una posible degradación. En los resultados, se puede observar la degradación del mancozeb en la cebolla: en el día 1, se halló 283,83ppb; el día 5 , 184,77ppb; en día 15, 182,4ppb y en el día 25, 177,44ppb, cantidades menores a $0,5 \mathrm{ppm}$ (límite máximo permisible para ditiocarbamatos, según el Codex Alimentarius (2013).

Cabe destacar que este método no ha sido utilizado antes en Colombia para la cuantificación de fungicidas en productos agrícolas, debido, principalmente, a que en el territorio nacional sólo se cuenta con tres equipos de este tipo, lo que resalta la importancia de este trabajo en el análisis de analitos y matrices, en este amplio campo, aportando resultados confiables y siendo un método sencillo y de bajo costo, pues se involucra solamente secado, extracción con solvente y dilución en solución Britton.

Se concluye, que las condiciones instrumentales y los principales parámetros de validación del método voltamétrico para la determinación cuantitativa de mancozeb en cebolla se establecieron, permitiendo obtener resultados válidos y confiables. La metodología desarrollada, se constituye en una alternativa económica y amigable con el entorno natural para aplicarse en cebolla cabezona, para la cuantificación del fungicida, objeto de estudio, pero además en otros cultivos, en los que se use este producto químico.

Agradecimientos: Los autores agradecen a Colciencias y a la Universidad Pedagógica y Tecnológica de Colombia, por el apoyo financiero al Convenio 0212-2013, titulado "Sistema de detección in situ de plaguicidas en la cadena de la papa al alcance de los agricultores". Conflictos de intereses: El manuscrito fue preparado y revisado con la participación de todos los autores, quienes declaramos que no existe conflicto de intereses, que ponga en riesgo la validez de los resultados presentados"

\section{BIBLIOGRAFÍA}

1. BLASCO, C.; FONT, G.; PICÓ, Y. 2004. Determination of dithiocarbamates and metabolites in plants by liquid chromatography-mass spectrometry. J. Chromatogr. A. 1028:267-276.
2. CODEX ALIMENTARIUS, 2013. Residuos de los plaguicidas en los alimentos y piensos. Disponible desde Internet en: http://www.codexalimentarius.net/pestres/data/commodities/details.html?id=265 (con acceso 20/09/2014).

3. DEARFIELD, K.L. 1994. Ethylene thiourea (ETU). A review of the genetic toxicity studies. Mutat. Res-Rev. Genet. 317(2):111-132.

4. GARCINUÑO, R.M.; FERNÁNDEZ-HERNANDO, P.; CÁMARA, C. 2004. Simultaneous determination of maneb and its main metabolites in tomatoes by liquid chromatography using diode array ultraviolet absorbance detection. J. Chromatogr. A. 1043(2):225229.

5. HARRISON, A.; CHOU, E.; GRAY, J.; POKIRWKA, N.; RALEY, K. 2013. Mancozeb-induced behavioral deficits precede structural neural degeneration. Neurotoxicology. 34:74-81.

6. KAUR, M.; KUMAR, A.; SINGH, B. 2011.Fourth derivative spectrophotometric method for the determination of fungicide maneb using sodium molybdate. Amer. J. Anal. Chem. 2: 158-163.

7. LÓPEZ, O.; RIAL, R.; SIMAL, J. 2013. Factors governing the removal of mancozeb residues from lettuces with washing solutions. Food Control. 34:530-538.

8. MINISTERIO DE AGRICULTURA. 2002. El cultivo de la cebolla cabezona blanca. Cartilla Didáctica. U.T. AGROTOM. p.3.

9. MUJAWAR, S.; UTTURE, S.; FONSECA, E.; MATARRITA, J.; BANERJEE, K. 2014. Validation of a GC-MS method for the estimation of dithiocarbamate fungicide residues and safety evaluation of mancozeb in fruits and vegetables. Food Chem. 150: 175-181.

10. PARAMASIVAM, M.; CHANDRASEKARAN, S. 2013. Dynamics and residues of mixed formulation of fenamidone and mancozeb in gherkin field ecosystem. Ecotox Environ Safe. 98: 292-296.

11. QIU, P.; NIAN, Y. 2008. Determination of ziram in vegetable samples by square wave voltammetry. Chinese Chemical Letters. 19, 1337-1340.

12. SENA. 2006. Curado y almacenamiento de cebolla de bulbo. Litolaser Editorial. Servicio Nacional de Aprendizaje SENA- Regional Cundinamarca. 6p. 
13. VALENTICH, M.A.; EYNARD, A.R.; BAROTTO, N.N.; DÍAZ, M.P.; BONGIOVANNI, G.A. 2006. Effect of the co-administration of phenobarbital, quercetin and mancozeb on nitrosomethylurea-induced pancreatic tumors in rats. Food Chem. Toxicol. 44: 2101-2105.

14. VETTORAZZI, G.; ALMEIDA, W.F.; BURIN, G.J.; JAEGER, R.B.; PUGA, F.R.; RAHDE, A.F.; REYES, F.G.; SCHVARTSMAN, S. 1995. International safety assessment of pesticides: dithiocarbamate pesticides, ETU, and PTU--A review and update. Teratog. Carcinog. Mutagen.15(6):313-317.
Recibido: Julio 31 de 2014

Aceptado: Septiembre 23 de 2014

\section{Como Citar:}

Martínez, E.R.; Dotor, M.Y.; Chaparro, S.P. 2014. Validación de un método voltamétrico para la cuantificación de mancozeb en cebolla cabezona (Allium cepa L.). Rev. U.D.C.A Act. \& Div. Cient. 17(2): 607-611. 